\title{
Efectos de las intenciones de implementación en las intervenciones sobre la salud*
}

\author{
Inge Schweiger-Gallo ${ }^{1}$, Jesús de Miguel ${ }^{2}$, Miryam RodrígueZ- \\ MONTER $^{1}$, JOsÉ-LuIS ÁLVARO ${ }^{1}$ Y PETER M. GOLLWITZER ${ }^{3}$ \\ ${ }^{1}$ Universidad Complutense de Madrid; ${ }^{2}$ Universidad Autónoma de Madrid; \\ ${ }^{3}$ New York University/University of Konstanz
}

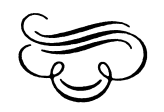

\section{Resumen}

En la última década, las intenciones de implementación han resultado ser estrategias eficaces de autorregulación en multitud de estudios (Gollwitzer y Sheeran, 2006). En este artículo revisaremos su eficacia en el campo de la Salud. Así, las intenciones de implementación han sido uitiles en la promoción de conductas saludables (por ejemplo, facilitación de pautas de dieta sana o realización de ejercicio físico), el seguimiento de metas no placenteras (auto-exploraciones o mediciones del nivel de glucosa en sangre, por ejemplo), en la facilitación del control de situaciones y circunstancias indeseadas, así como de las acciones encaminadas hacia una meta en aquellas poblaciones críticas (esquizofrénicos, niños con trastorno por déficit de atención con hiperactividad, etc.) que tienen dificultades en el control de la acción. Que la efectividad de las intenciones de implementación no está además acompañada de rigidez y no produce efectos de rebote avala la importancia de esta estrategia de autorregulación. Se discuten las limitaciones de las intenciones de implementación, así como futuras intervenciones mediante esta estrategia.

Palabras clave: Intenciones de implementación, metas, salud, intervenciones.

\section{Effects of implementation intentions on health interventions}

\begin{abstract}
In the past decade, implementation intentions have consistently been shown to be effective self-regulatory tools in a multitude of studies (Gollwitzer E Sheeran, 2006). In this article, we will review their effectiveness in the bealth domain. Thus, by forming implementation intentions it is possible to foster the promotion of healthy (healthy eating, physical exercise) and unpleasant behaviors (bome blood glucose monitoring or self-examinations), as well as the control of unwanted influences and the promotion of goal attainment in critical populations that have difficulties with action control (such as schizophrenics and children with ADHD). Moreover, recent research on the costs of action control by implementation intentions has also revealed that forming implementation intentions is not accompanied by rigidity, or by rebound effects, which points to the importance of this self-regulatory strategy. The limitations of this strategy, as well as future interventions are discussed.

Keywords: Implementation intentions, goal intentions, health, interventions.
\end{abstract}

*Una versión preliminar de este artículo ha sido publicada en la edición electrónica de las Actas del X Congreso Nacional de Psicología Social celebrado en Cádiz en 2007.

Correspondencia con los autores: Inge Schweiger Gallo, Departamento de Psicología Social, Facultad de Ciencias Políticas y Sociología, Campus de Somosaguas, 28223 Pozuelo de Alarcón (Madrid). Teléfono 9139427 66. Fax 9139430 29. Email: ingesg@cps.ucm.es 
Es de sobra conocido que la intención constituye un concepto clave en la Psicología. Así se pone de manifiesto en numerosas investigaciones como, por ejemplo, las derivadas de la teoría de la conducta planeada o la de la acción razonada (p. ej., Ajzen y Madden, 1986; Madden, Ellen y Ajzen, 1992). Sin embargo, aunque se ha prestado mucha atención a la relación entre actitud y conducta, éste no ha sido el caso respecto a los mecanismos psicológicos que median los efectos de las intenciones sobre el comportamiento (Gollwitzer, Bayer y McCulloch, 2005). Esto es tanto más llamativo si tenemos en cuenta los resultados que señalan que las intenciones sólo explican un escaso porcentaje de la varianza de futuras conductas (Sheeran, 2002). Esta brecha entre las intenciones y la conducta actual es comúnmente reducida cuando tomamos en consideración la formación de intenciones de implementación (Gollwitzer y Sheeran, 2006).

Tal y como veremos en el presente artículo, las intenciones de implementación han resultado ser estrategias más eficaces que las metas simples para promover comportamientos como la toma diaria de medicamentos (e.g., Sheeran y Orbell, 1999) o cuando hay que realizar acciones no placenteras como acudir a una citología (Sheeran y Orbell, 2000). Asimismo, han demostrado facilitar las acciones encaminadas hacia una meta en poblaciones críticas, como pacientes con lesiones cerebrales frontales (Lengfelder y Gollwitzer, 2001) o con esquizofrenia y pacientes bajo el síndrome de abstinencia (Brandstätter, Lengfelder y Gollwitzer, 2001), para quienes los problemas de iniciación de acciones dirigidas hacia una meta están más acentuados.

A pesar de la importancia de estas estrategias, en la Psicología hispana e hispanoamericana apenas se les ha otorgado importancia y se les ha prestado una escasa atención, aún cuando desde hace más de una década ocupan un lugar central dentro de la Psicología internacional y son objeto de numerosas publicaciones en revistas del calado de Journal of Personality and Social Psychology, el Journal of Experimental Social Psychology, o el European Journal of Social Psychology. De ahí que presentemos esta revisión de la eficacia de las intenciones de implementación en un campo específico, la Psicología de la Salud.

\section{La diferenciación entre metas simples e intenciones de implementación}

Las intenciones de implementación forman parte de las teorías de autorregulación de la consecución de metas que intentan explicar los procesos volitivos implicados en dicha consecución (Gollwitzer y Moskowitz, 1996). En este contexto, la autorregulación puede verse comprometida por cuatro problemas identificados como críticos para la consecución de las metas (Gollwitzer y Sheeran, 2006). Se trata, en primer lugar, de resolver el problema de iniciar las conductas especificadas en las metas propuestas, es decir, de aprovechar buenas oportunidades para actuar (e.g., para tomar pastillas regularmente; Sheeran y Orbell, 1999). Asimismo, una vez iniciada la conducta, esta ha de ser mantenida, persistiendo frente a distracciones, dificultades e interferencias (e.g., regular reacciones emocionales indeseadas; Schweiger Gallo, Keil, McCulloch, Rockstroh y Gollwitzer, 2009). En tercer lugar, puede ser necesario cambiar de meta (p. ej., cambiando a medios más efectivos; Henderson, Gollwitzer y Oettingen, 2007), así como, finalmente, preservar recursos para otras metas (prevenir, por ejemplo, el efecto de egodepletion; Webb y Sheeran, 2003). Intentar paliar estos problemas en la consecución de metas es uno de los principales objetivos de la teoría de las intenciones de implementación (Gollwitzer, 1993, 1999).

Las intenciones de implementación (implementation intentions) son planes "si-entonces" ("if-then plans") que especifican cuándo, dónde y cómo se va a llevar a la acción una meta propuesta: "Si me encuentro con la situación $x$, entonces llevaré a cabo la conducta $y$ ", uniendo de esta manera una situación crítica con una conducta dirigida a la meta. Peter Gollwitzer las distingue de las metas simples (goal intentions): metas que aluden a un comportamiento o resultado deseado y tienen el formato de "Voy a intentar alcanzar $z$ ". Mientras que las metas simples designan estados finales deseados con cuyo logro el indi- 
viduo se siente comprometido, las intenciones de implementación se refieren a la realización de la meta simple. De hecho, las intenciones de implementación crean un compromiso de responder a la situación crítica especificada (parte "si") de una manera planeada, dirigida hacia la meta (parte "entonces"). Las intenciones de implementación están subordinadas jerárquicamente a las metas simples, de manera que están formadas en función de éstas. Dicho de otra forma, mientras que las metas simples suponen un deseo, las intenciones de implementación constituyen planes articulados para conseguir tales metas (o meta), delimitando un plazo de tiempo (cuándo), un escenario (dónde) y una estrategia (cómo). Las intenciones de implementación se subordinan de esta manera a la meta simple, al igual que todo plan solo cobra sentido en función del objetivo que persigue.

La eficacia de las intenciones de implementación se fundamenta en dos tipos de procesos: los relacionados con la situación anticipada, y aquellos que remiten a la conducta especificada (Gollwitzer, 1993, 1999). En cuanto a la situación anticipada, las investigaciones de los últimos años han demostrado que las intenciones de implementación crean un vínculo mental entre la situación crítica especificada y la conducta intencionada orientada a la meta. En consecuencia, la representación mental de la situación crítica se activa y es por tanto altamente accesible. Esto permite una detección fácil, un recuerdo efectivo, así como una disposición a atender a la situación crítica incluso estando ocupado.

Por lo que se refiere a la conducta especificada, la formación de un vínculo "si-entonces" delega el control de la conducta dirigida hacia la meta a la situación crítica. En consecuencia, se produce una automatización de la iniciación de los esfuerzos dirigidos a la meta en presencia de la situación especificada, siendo dicha iniciación de la acción inmediata (Gollwitzer y Brandstätter, 1997, Estudio 3), eficiente (Brandstätter et al., 2001) y sin requerir un intento consciente (Sheeran, Webb y Gollwitzer, 2005, Estudio 2). Dicho control automático de la acción también ha sido denominado "automaticidad estratégica" o "hábitos instantáneos", dado que dichos procesos automáticos están fundamentados en un solo acto mental basado en unir una situación crítica con una conducta deseada dirigida hacia una meta (Gollwitzer y Schaal, 1998) y no es resultado de una conducta pasada habitual, como sucede con los hábitos (Aarts y Dijksterhuis, 2000).

\section{Las intervenciones mediante intenciones de implementación}

En el ámbito de la salud nos encontramos con gran cantidad de conductas saludables que no son llevadas a cabo debido a que no se logra siquiera iniciar dicha conducta. Sheeran y Orbell (1999) y Bermúdez, Contreras, Margüenda y García (2004), por ejemplo, analizaron si las intenciones de implementación podían promover la consecución de una conducta: la toma diaria de vitaminas. Tanto los dos estudios citados de Sheeran y Orbell como el de Bermúdez y colaboradores constataron que los participantes que se formaban una intención de implementación se olvidaban de menos pastillas tras varias semanas ( 3 semanas en el caso de Sheeran y Orbell, y 4 semanas en el de Bermúdez y colaboradores) que aquellos participantes con una meta simple.

Son varios también los estudios que han analizado la efectividad de las intenciones de implementación sobre una alimentación saludable, tanto en niños como adultos (e.g., de Nooijer, de Vet, Brug y de Vries, 2006; Gratton, Povey y Clark-Carter, 2007; Sullivan y Rothman, 2008). Armitage (2004), por ejemplo, evaluó los efectos de las intenciones de implementación sobre la reducción de la ingesta de grasas en una muestra de trabajadores de una empresa. Un mes después de la intervención, sólo se había reducido la ingesta de grasa del grupo experimental que se formó una intención de implementación, tal y como demostraban tres índices diferentes de ingesta de grasa. La efectividad de estas instrucciones se analizó durante un plazo mayor gracias a Luszczynska, Scholz y Sutton en el año 2007. Estos autores observaron en un grupo de pacientes que había sufrido un infarto de miocardio que sólo aquellos que se habían formado una intención de implementación fueron capaces de reducir su ingesta de grasas durante 6 meses. 
Otro tema recurrente en la Psicología de la Salud lo constituye la realización de ejercicio físico. No sorprende, pues, que también en este ámbito se hayan aplicado las intenciones de implementación en un número elevado de intervenciones, tanto con pacientes, como con población normal (e.g., Milne, Orbell y Sheeran, 2002; Rise, Thompson y Verplanken, 2003; Walsh, da Fonseca y Banta, 2005). Prestwich, Lawton y Conner (2003), por ejemplo, pidieron a los participantes que realizaran ejercicio físico dos veces más a la semana del que ya realizaban sin reducir el tiempo dedicado a cada sesión. Tal y como predijeron, tras cuatro semanas observaron un incremento en la frecuencia y el tiempo total dedicado al ejercicio físico, así como una mejora del estado físico en aquellos participantes que se formaron una intención de implementación. Estos datos cobran especial importancia dado que se basan en una combinación de datos subjetivos con medidas objetivas, como la tasa cardiaca media.

Un tipo específico de ejercicio es aquel que han de llevar a cabo las personas que se han sometido recientemente a una operación quirúrgica, se encuentran en rehabilitación o a los que se recomienda ejercitarse debido a alguna dolencia. Aplicado a una muestra de pacientes que habían sufrido recientemente un infarto de miocardio, Luszczynska (2006) encontró que aquéllos que se formaban intenciones de implementación y recurrían frecuentemente a la estrategia de planificar, llevaron a cabo durante seis meses la actividad física moderada recomendada. Es conveniente señalar que en este estudio las intenciones de implementación se formaron en presencia del experimentador, que no sólo proveía al paciente de retroalimentación, sino que también permitía ajustar los planes para que éstos fueran específicos y realistas. En lo referente a pacientes en rehabilitación ortopédica, Ziegelmann, Luszczynska, Lippke y Schwarzer (2007) llevaron a cabo un estudio longitudinal en el que constataron cómo -tanto a los 6 como a los 12 meses- los participantes en el grupo de intención de implementación llevaban a cabo en mayor medida ejercicios físicos que el grupo de control.

Otro ámbito en el que son relevantes las intervenciones mediante intenciones de implementación es el de la seguridad laboral, así como la asistencia a psicoterapia. Sheeran y Silverman (2003) aplicaron esta estrategia con el objetivo de incrementar las tasas de asistencia a los cursos de entrenamiento de seguridad en caso de incendios. Las tasas de asistencia demostraron que aquellos participantes que se habían formado una intención de implementación acudieron en mayor número a dichos cursos (39\%) que aquellos participantes que habían recibido un mensaje simplemente dirigido a incrementar su motivación (12\%) o de los participantes en la condición de control (16\%). Asimismo, hay que destacar que no se encontraron diferencias significativas cuando se analizó si los participantes acudían en los primeros 20 días tras recibir la instrucción o hasta 64 días más tarde, lo que indica que las intenciones de implementación fueron eficaces durante todo ese plazo. Igualmente, Sheeran, Aubrey y Kellett (2007) observaron que gracias a la formación de una intención de implementación se incrementaba, por un lado, la intención de tratarse (es decir, de acudir a psicoterapia) y, en segundo lugar, el número de personas que efectivamente acudían a psicoterapia.

Asimismo, se ha analizado el seguimiento de metas no placenteras, tales como la realización de citologías (Sheeran y Orbell, 2000) o mediciones del nivel de glucosa en sangre (Liu y Park, 2004). Más específicamente, Sheeran y Orbell (2000) pudieron mostrar que mientras que la inmensa mayoría de las participantes en la condición de intención de implementación (92\%) acudió a sus citas en un intervalo de tres meses, las participantes que se formaron una meta simple acudieron en un porcentaje significativamente menor $(69 \%)$ a realizarse una citología. Es más, los resultados obtenidos indican que las intenciones de implementación redujeron el hábito de demora anterior. La aplicación de las intenciones de implementación también ha arrojado resultados interesantes en cuanto a la realización de auto-exploraciones testiculares (Steadman y Quine, 2004), dado que la realización de éstas se vio significativamente aumentada en un periodo que abarcó 3 semanas en aquellos participantes que se habían formado una intención de imple- 
mentación (65\%) en comparación con los participantes del grupo de control (40\%). Varios estudios (e.g., Rutter, Steadman y Quine, 2006) han analizado igualmente cómo fomentar la detección temprana de enfermedades de mama, ya sea mediante mamografías o mediante auto-exploraciones de mama. La eficacia de las intenciones de implementación, de hecho, ya había sido constatada en este terreno en un artículo del año 1997 firmado por Orbell, Hodgkins y Sheeran. Tras un mes, el 64\% de las mujeres del grupo de intervención habían realizado auto-exploraciones, mientras que sólo fue el caso por parte del $14 \%$ de las participantes del grupo de control. Posteriormente, Prestwich et al. (2005) contrastaron estos efectos en sendos estudios durante un plazo de tiempo mayor. En el primer estudio, la probabilidad de llevar a cabo una auto-exploración de mamas se incrementaba tanto al mes como a los 6 meses de la intervención. Asimismo, el número de auto-exploraciones realizadas fue mayor en ambos momentos por parte de aquellas participantes que se habían formado una intención de implementación. En un segundo estudio, estos investigadores incluyeron una medida adicional: intenciones de implementación que no estaban basadas meramente en la planificación individual, sino que incluían a la pareja como variable social. Así, las participantes decidían junto a su pareja cuándo y dónde la pareja iba a realizar la exploración de mamas. Los resultados mostraron que ésta última estrategia fue especialmente efectiva.

Comparativamente pocos han sido los estudios que se han centrado en el segundo de los problemas de autorregulación: el mantenimiento de la conducta, una vez iniciada ésta. Una excepción reciente la constituye una serie de experimentos que han analizado la efectividad de las intenciones de implementación para proteger los esfuerzos dirigidos a alcanzar una meta frente a estímulos externos e internos. Achtziger, Gollwitzer y Sheeran (2008; Estudio 1) aplicaron esta estrategia para ayudar a los participantes a evitar picar comida entre horas. Conforme a las hipótesis, los participantes que se formaron una intención de implementación lograron evitar los antojos, asegurando así la meta de hacer dieta.

Tampoco debemos de olvidarnos de aquellas intervenciones destinadas a evitar conductas que hacen peligrar o comprometen la salud. Un ejemplo lo constituye el artículo firmado por Armitage en 2007. En él, Armitage (2007b) describe una intervención basada en la utilización de intenciones de implementación como herramienta para dejar de fumar. Tras 2 meses, la dependencia de nicotina fue menor en aquellos que habían recibido la intervención basada en la intención de implementación. Es más, también el porcentaje de personas que dejaron de fumar aumentó en comparación con el grupo de control.

Por último, la promoción de la consecución de metas en aquellas poblaciones que tienen dificultades en el control de la acción también se ha visto significativamente incrementada gracias a la formación de intenciones de implementación. Este ha sido el caso de pacientes con lesiones cerebrales frontales (Lengfelder y Gollwitzer, 2001), esquizofrenia o con adicciones a los opiáceos bajo el síndrome de abstinencia (Brandstätter et al., 2001), así como niños con trastorno por déficit de atención con hiperactividad (Gawrilow y Gollwitzer, 2008). Brandstätter et al. (2001), por ejemplo, pidieron a un grupo de pacientes esquizofrénicos y a un grupo de control que llevasen a cabo una tarea go/no-go tras recibir una intención de implementación ("Si aparece el número 3, voy a responder especialmente rápido") o la meta de acelerar la velocidad de respuesta al número 3 familiarizándose con la instrucción (por ejemplo, escribiendo repetidamente el número en una hoja de papel). Los resultados mostraron que los pacientes que se formaron una intención de implementación aumentaron su velocidad de respuesta con respecto a los estímulos críticos, pero no a los no-críticos; todo ello, a pesar del déficit que presentan éstos enfermos en cuanto a la atención selectiva y a pesar de la mayor vulnerabilidad a distracciones que los caracterizan. Otro grupo de población que se ha beneficiado de la eficacia de las intenciones de implementación son los niños con trastorno por déficit de atención con hiperactividad. En dos estudios publicados en 2008, Gawrilow y 
Gollwitzer encontraron que sólo aquellos niños que añadieron una intención de implementación a una meta simple para inhibir respuestas indeseadas, fueron capaces de perseguir su meta de inhibir una respuesta en comparación con aquellos niños que sólo se habían formado una meta simple. Asimismo, se ha analizado si esta estrategia ayuda a suprimir respuestas emocionales negativas, tales como el asco y el miedo (Schweiger Gallo et al, 2009). Los resultados avalaron la eficacia de las intenciones de implementación dado que sólo los participantes que se propusieron una intención de implementación fueron capaces de controlar su asco (Estudio 1) y miedo (Estudios 2 y 3). Es más, los participantes, que contaban previamente con un acusado miedo a las arañas, fueron capaces de controlar su miedo reduciéndolo incluso al mismo nivel que los participantes sin miedo a estos arácnidos.

\section{¿Conllevan costes las intenciones de implementación?}

En lo concerniente a los posibles costes de formar intenciones de implementación, estudios recientes han podido mostrar que la efectividad de las intenciones de implementación no está acompañada de rigidez y no produce efectos de rebote ni ego-depletion, lo que avala la importancia de esta estrategia de autorregulación. De hecho, Webb y Sheeran observaron en 2003 que la consecución de una meta gracias a la formación de intenciones de implementación se logra sin incurrir en consecuencias negativas tales como el efecto de ego-depletion (Baumeister, Bratslavsky, Muraven y Tice, 1998). Esto indica que el alcance de la meta propuesta mediante esta estrategia de autorregulación deja intactas las capacidades de autorregulación de las personas, dado que los participantes que se formaron una intención de implementación para llevar a cabo una tarea difícil como lo era tener un buen rendimiento en la tarea Stroop, no mostraron déficit alguno en la regulación con éxito de una tarea cognitiva posterior. Las consecuencias cognitivas de la formación de intenciones de implementación sobre el control del miedo también fueron objeto de estudio por parte de Schweiger Gallo y Gollwitzer (2007). Los resultados mostraron que, a pesar de que todos los participantes evaluaron imágenes bajo una carga cognitiva, sólo aquellos que se propusieron una intención de implementación tuvieron unas reacciones emocionales menos acentuadas en respuesta a las imágenes de arañas que aquellos que se propusieron una meta simple o los participantes que no tenían meta alguna. Así, el control de las emociones mediante intenciones de implementación no afectó a los recursos cognitivos de los participantes, lo que avala la automaticidad de esta estrategia de autorregulación.

Otra evidencia empírica que muestra que las intenciones de implementación no producen costes fue aportada por Gollwitzer, Trötschel, Bayer y Sumner (citados en Gollwitzer et al., 2005). En sendos estudios mostraron que las intenciones de implementación utilizadas para el control de estereotipos no producían efectos de rebote. Los participantes tuvieron que formarse en primer lugar una impresión no estereotípica de una persona sin hogar antes de evaluar a personas sin hogar en general (Estudio 1) o llevar a cabo una tarea de decisión léxica que evaluaba la accesibilidad del estereotipo de persona sin hogar (Estudio 2). En comparación con los participantes que añadieron a su meta simple una intención de implementación, los juicios de los participantes de la condición de meta simple fueron más estereotípicos en el primer estudio. Asimismo, éstos últimos participantes identificaron con mayor rapidez los estereotipos en el segundo estudio, desvelando una mayor accesibilidad del estereotipo de persona sin hogar.

\section{Limitaciones de las intervenciones mediante intenciones de implementación}

Si bien la eficacia de las intervenciones mediante intenciones de implementación está avalada ampliamente (véase el meta-análisis de Gollwitzer y Sheeran del año 2006), también nos encontramos con estudios en los que las intenciones de implementación no han contribuido significativamente a fomentar una conducta saludable o lo han hecho 
tras un período de tiempo. Un ejemplo lo representa el estudio de Lavin y Groarke del año 2005. Estos autores analizaron si formar intenciones de implementación contribuiría a utilizar seda dental con mayor frecuencia. En contra de lo que cabría esperarse, no encontraron diferencias significativas durante las 3 semanas que duró el estudio entre el grupo de intervención que recibió la instrucción de intención de implementación y el grupo de control. Otro estudio de Higgins y Conner (2003) no obtuvo resultados significativos en un estudio centrado en la prevención del tabaquismo, aunque la tendencia de las intenciones de implementación iba en la dirección prevista. Como reconocen los propios autores, la falta de datos estadísticamente significativos ha de ser atribuida a una muestra demasiado pequeña, así como a unos intervalos de medición demasiado cortos.

A pesar de que encontramos, como hemos expuesto, numerosos estudios que avalan la eficacia de las intenciones de implementación sobre la realización de ejercicio físico, queda por reseñar que algunos autores como Budden y Sagarin (2007) observaron que las intenciones de implementación no fueron efectivas en el caso de sus muestras. De hecho, aquellos participantes en el estudio de Budden y Sagarin que especificaron el cuándo, dónde y cómo hacer ejercicio durante la semana siguiente no sólo se ejercitaron menos, sino que aquellos que no las formaron lo hicieron en mayor medida. Entre las explicaciones que barajan los autores se encuentran la existencia de diferencias individuales o bien una excesiva rigidez de las instrucciones.

Otro estudio (Jackson et al., 2005) investigó si las intenciones de implementación servían para que las personas aumentasen el número de piezas de frutas y verduras que comían al día. Transcurridos 7, 28 y 90 días, la ingesta no se diferenció entre los diferentes grupos. Varias son las explicaciones ofrecidas por los autores para esta pauta de resultados: en primer lugar, las intenciones de implementación especificaron cuando, qué y dónde iban a tomar la pieza de fruta o verduras, pero no dónde adquirirla. Asimismo, hay que tener en cuenta que la muestra no estaba compuesta de estudiantes, sino de pacientes motivados en cambiar sus pautas alimenticias, y que éstos pudieron formar intenciones de implementación espontáneas. Según los autores, también pudieron diferir en el tiempo las condiciones especificadas en la intención de implementación.

Jackson et al. (2006) quisieron comprobar si las intenciones de implementación ayudaban a que un grupo de pacientes tomara el antibiótico recetado sin olvidarse de la dosis o dando por acabado el tratamiento antes de tiempo. Los resultados mostraron que la gran mayoría de los pacientes (76\%) tómo la medicación si bien no encontraron diferencias entre los diferentes grupos. Independientemente de las explicaciones ofrecidas por Jackson y colaboradores (formación de intenciones de implementación espontáneas por parte de los otros participantes; cambios de hábito para tomar el antibiótico que excedían el contenido especificado en las intenciones de implementación, uso de población no estudiantil), tal y como sugieren, la ausencia de eficacia de estos estudios se puede deber a que se trata de conductas simples que se llevan a cabo a corto plazo, por lo que no resulta necesario formarse planes para alcanzarlas (Gollwitzer y Brandstätter, 1997). De hecho, las investigaciones sobre intenciones de implementación han venido mostrando que estas estrategias resultan más eficaces cuanto más difícil sea de alcanzar la conducta, así como en poblaciones problemáticas (por ejemplo, enfermos de esquizofrenia; Brandstätter et al., 2001).

En general, carecemos aún de evidencia empírica que nos ayude a dilucidar el por qué de la ausencia de efectos en algunos estudios. Un denominador común de muchos de estos trabajos parece ser la variable temporal. Mientras que algunas intervenciones parecen surtir mayor efecto transcurrido un cierto tiempo (e.g., la intervención de Sheeran y Orbell de 1999 surtió efectos pasadas 3 semanas, pero no tras 10 días, lo que concuerda con la pauta observada por Sullivan y Rothman en 2008, según la cual los efectos de las intenciones de implementación aumentan conforme pasa el tiempo), en otras se ha encontrado una pauta inversa (en el primer estudio de Prestwich y colaboradores de 
2005, por ejemplo, las intenciones de implementación, si bien efectivas, lo fueron ligeramente en menor medida tras seis meses que tras 1 mes) y en otros, aún tratándose de la misma conducta (ingesta de pastillas de vitaminas, como en el estudio de Sheeran y Orbell, de 1999) surgen los efectos tras pocos días y se mantienen durante semanas (Bermúdez et al., 2004). Dadas estas pautas contradictorias no podemos deducir que la efectividad de las intenciones de implementación sea mayor en el caso de promoción de conductas que en el mantenimiento de dichas conductas. Sin duda alguna, necesitamos de un mayor número de estudios longitudinales que complementen los datos con los que contamos a día de hoy.

Tampoco hemos de olvidarnos del papel fundamental que juegan diferentes moderadores sobre los efectos de las intenciones de implementación (Sheeran, Milne, Webb y Gollwitzer, 2005). Como ya hemos expuesto anteriormente, el empleo de intenciones de implementación puede resultar superfluo en el caso de problemas de auto-regulación fácilmente solubles. También es imprescindible la existencia de una meta supraordenada fuerte, así como tener en cuenta el grado de formación de la intención de implementación: así queda evidenciado en un reciente estudio firmado por Rutter et al. (2006). En él, la realización de una mamografía sólo se diferenció entre las diversas condiciones cuando se seleccionaron sólo a aquellas participantes que habían seguido las instrucciones, es decir, cumplimentado en los cuestionarios los planes específicos para llevar a cabo la conducta. De ahí que, a falta de contrastar si la ausencia de resultados significativos era debida a la presencia de estos moderadores o a la contrastada eficacia de estas instrucciones, éstos resultados deben ser tomados aún con cautela.

\section{Futuras intervenciones mediante intenciones de implementación}

Como se desprende de lo expuesto hasta el momento, las intenciones de implementación han sido aplicadas principalmente desde un enfoque basado en la promoción de la salud. Así, hemos visto que contamos con numerosos estudios basados en la facilitación de pautas de dieta sana (e.g., de Nooijer et al., 2006), de la realización de ejercicio físico (p. ej., Prestwich et al., 2003), o la realización de chequeos médicos (e.g., Steadman y Quine, 2004). Sin embargo, aún quedan ámbitos dentro de la Psicología de la Salud (ver Morrison y Bennett, 2008) en los que apenas se ha aplicado la implementación de intenciones, como es el caso de la obesidad. Teniendo en cuenta que una de las metas principales de la Psicología de la Salud no es otra que poner freno a la ejecución de conductas que comprometan la salud, no debemos de olvidarnos tampoco de conductas tales como el consumo de drogas. Asimismo, en comparación con los numerosos estudios que analizan cómo fomentar una alimentación saludable o la realización de ejercicio, sorprende la escasa importancia concedida a la prevención de conductas sexuales de riesgo. Mientras que ésta ocupa un lugar importante y tradicional en la Psicología (ver por ejemplo Sheeran y Orbell, 1998), apenas ha sido objeto de investigación ni intervención mediante intenciones de implementación hasta el momento.

En todo caso, las investigaciones futuras que se basen en la utilización de las intenciones de implementación no debieran de perder de vista la necesidad de utilizar medidas no basadas exclusivamente en la auto-evaluación. De hecho, aún son relativamente escasos los estudios que utilizan medidas objetivas como el número de cursos a los que se ha asistido (Sheeran y Silverman, 2003), el número de citologías realizadas (Sheeran y Orbell, 2000), asistencia a la cita de psicoterapia (Sheeran $e t$ al., 2007) o el estado físico tras la intervención (Prestwich $e t$ al., 2003). No es desdeñable tampoco el número de estudios que se han llevado a cabo en este área basados en muestras estudiantiles (ver Tabla I). Aunque esta pauta está cambiando paulatinamente, sobre todo en los últimos años, aún se requieren investigaciones que apliquen las intenciones de implementación a colectivos que sean objeto de intervenciones, así como a poblaciones específicas. 


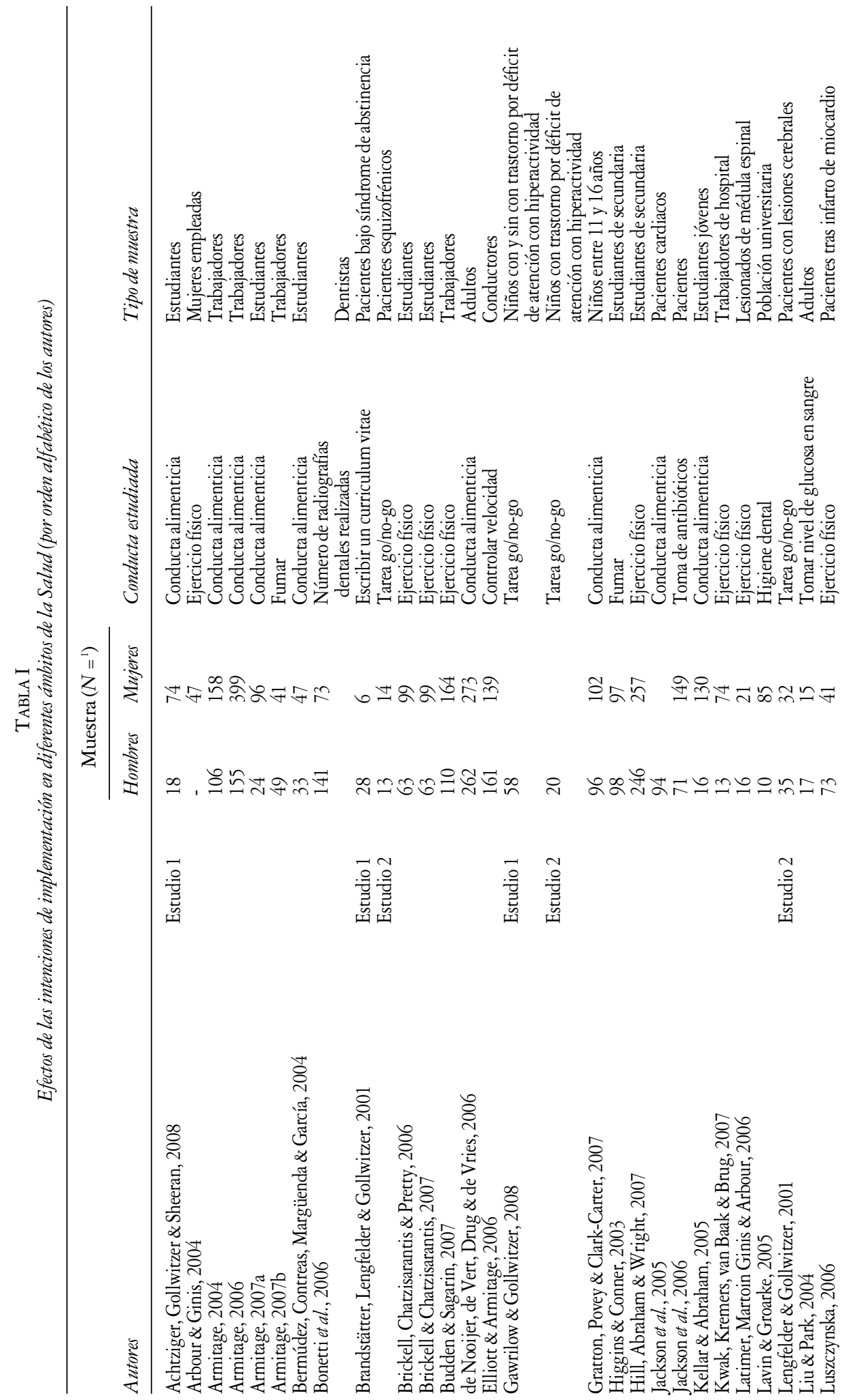




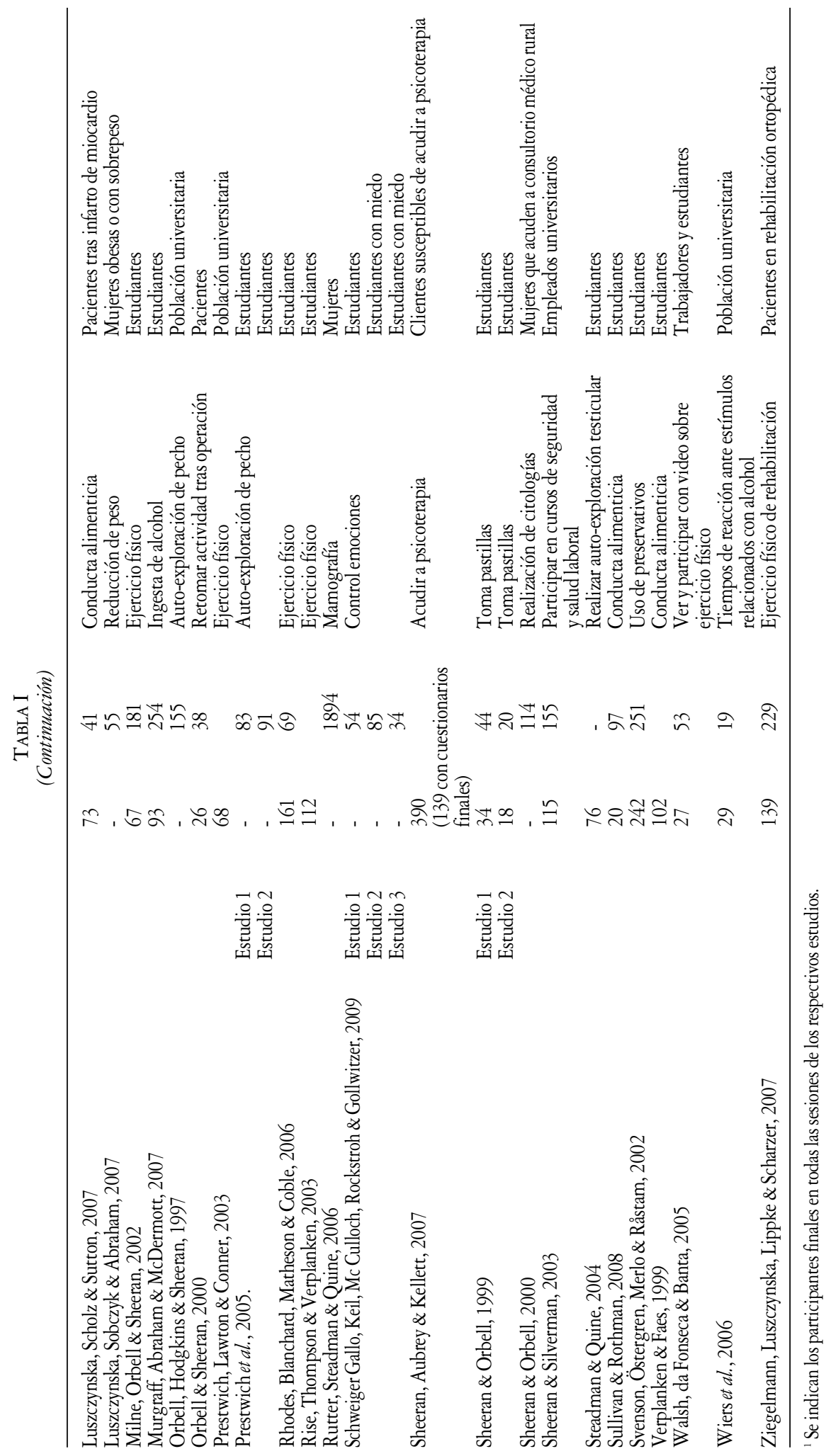


En efecto, y siguiendo las investigaciones expuestas, las intenciones de implementación han resultado ser eficaces para pacientes con esquizofrenia y adictos a los opiáceos bajo el síndrome de abstinencia (Brandstätter et al., 2001). También pacientes con lesiones cerebrales frontales (Lengfelder y Gollwitzer, 2001) o niños con trastorno por déficit de atención con hiperactividad (Gawrilow y Gollwitzer, 2008) tuvieron éxito en promover sus comportamientos dirigidos hacia una meta tras añadir a su meta simple una intención de implementación. Pero las intenciones de implementación también podrían ayudar a otros colectivos. Así, por ejemplo, los pacientes depresivos podrían aprovecharse de esta estrategia para formar planes que especifiquen cómo realizar tareas concretas. Progresando hacia las diferentes metas, la capacidad de autoeficacia de estas personas podría aumentar, facilitando una reincorporación a una vida normalizada. Las intervenciones mediante intenciones de implementación debieran de ser asimismo particularmente valiosas tanto para los profesionales que tienen que manejar a diario sensaciones severas de asco (e.g., profesionales de la salud), como para aquellos otros con comportamientos obsesivo-compulsivos, que debieran de sacar provecho de formar intenciones de implementación para compensar su dificultad o incapacidad para suprimir pensamientos indeseados y disfuncionales (Wenzlaff y Wegner, 2000). De hecho, formar planes "si-entonces" podría complementar intervenciones clínicas tradicionales de larga duración (e.g., desensibilización sistemática) que aspiran a facilitar el control de emociones negativas. Asimismo, este simple acto voluntario también podría incrementar el bienestar y aumentar la calidad de vida de las personas que han sido víctimas de traumas causados por la violencia instrumental.

\section{Conclusión}

A lo largo de las últimas décadas, las investigaciones han ido mostrando cómo la formulación de metas, incluso siendo éstas altamente deseables y viables, no es suficiente para su consecución. Para solventar precisamente los principales problemas de autorregulación (tales como iniciar la conducta especificada y/o mantenerla frente a distracciones, dificultades e interferencias), Gollwitzer $(1993,1999)$ propone recurrir a intenciones de implementación. Se trata de planes "si-entonces" que especifican cuándo, dónde y cómo se van a llevar a cabo las acciones encaminadas a conseguir la meta propuesta.

La eficacia de las intenciones de implementación queda avalada por la gran cantidad de publicaciones sobre el tema. De hecho, el número de investigaciones dedicadas a intervenciones mediante intenciones de implementación en el ámbito de la salud ha crecido exponencialmente en los últimos años. A pesar de algunos artículos en los que no surtieron efecto -escasos, en comparación con los 94 estudios analizados en el metaanálisis de Gollwitzer y Sheeran (2006)-, podemos concluir que no solo la investigación básica motivacional y de autorregulación, sino también la investigación en el ámbito clínico de la salud se ha visto beneficiada a lo largo de la última década por los trabajos expuestos en este artículo. No obstante, la evidencia acumulada que muestra que la introducción del concepto de intención de implementación ha permitido incrementar sustancialmente la probabilidad de llevar a cabo determinadas conductas no es óbice para que aún se requiera de un número considerable de trabajos que contrasten la eficacia, pero también las limitaciones que pueden acompañar a dichas estrategias en diferentes ámbitos y con diferentes poblaciones, así como con diferentes medidas y en diferentes intervalos temporales.

Las implicaciones de la formación de esta estrategia están claras: no es necesario ningún entrenamiento para formarlas (Gollwitzer y Sheeran, 2006) y debido al control automático de la acción instigado por las intenciones de implementación, no se consumen recursos cognitivos. Es más, en lo concerniente a los posibles costes de formar intenciones de implementación, estudios recientes han podido mostrar que su efectivi- 
dad no está acompañada de rigidez, y no produce efectos de rebote ni ego-depletion, lo que avala la importancia de esta estrategia de autorregulación.

\section{Referencias}

Aarts, H. \& Dijksterhuis, A. (2000). Habits as knowledge structures: Automaticity in goal-directed behavior. Journal of Personality and Social Psychology, 78, 53-63.

ACHTZIGer, A., Gollwitzer, P. M. \& SHeEran, P. (2008). Implementation intentions and shielding goal striving from unwanted thoughts and feelings. Personality and Social Psychology Bulletin, 34, 381-393.

AJZEN, I. \& MADDEN, T. J. (1986). Prediction of goal-directed behavior: Attitudes, intentions, and perceived behavioral control. Journal of Experimental Social Psychology, 22, 453-474.

Arbour, K. P. \& GiNIS, K. A. M. (2004). Helping middle-aged women translate physical activity intentions into action: Combining the theory of planned behavior and implementation intentions. Journal of Applied Biobehavioral Research, 9, $172-187$.

ArmitAge, C. J. (2004). Evidence that implementation intentions reduce dietary fat intake: A randomized trial. Health Psychology, 23, 319-323.

ARMITAGE, C. J. (2006). Evidence that implementation intentions promote transitions between the stages of change. Journal of Consulting and Clinical Psychology, 74, 141-151.

Armitage, C. J. (2007a). Effects of an implementation intention-based intervention on fruit consumption. Psychology $\mathcal{E}$ Health, 22, 917-928.

ARMITAGE, C. J. (2007b). Efficacy of a brief worksite intervention to reduce smoking: The roles of behavioral and implementation intentions. Journal of Occupational Health Psychology, 12, 376-390.

Baumeister, R. F., BRATSLaVSKy, E., MuraVen, M. \& TICE, D. M. (1998). Ego depletion: Is the active self a limited resource? Journal of Personality and Social Psychology, 74, 1252-1265.

Bermúdez, J., Contreras, A., Margǘnda, A. \& García, A. M. P. (2004). Procesos motivacionales y volitivos en el desarrollo de conducta saludable: Utilidad diferencial de la implementación de la intención/ Motivational and volitional processes in the development of health behavior: Differential usefulness of implementation intentions. Análisis y Modificación de Conducta, 30, 71-103.

Bonetti, D., Pitts, N. B., Eccles, M., Grimshaw, J., Johnston, M., Steen, N. et Al. (2006). Applying psychological theory to evidence-based clinical practice: Identifying factors predictive of taking intra-oral radiographs. Social Science $\mathcal{E}$ Medicine, 63, 1889-1899

BRANDSTÄTtER, V., LENGFELDER, A. \& GOLLWITZER, P. M. (2001). Implementation intentions and efficient action initiation. Journal of Personality and Social Psychology, 81, 946-960.

Brickell, T. A., Chatzisarantis, N. L. D. \& Pretty, G. M. (2006). Using past behaviour and spontaneous implementation intentions to enhance the utility of the theory of planned behaviour in predicting exercise. British Journal of Health Psycho$\log y, 11,249-262$.

Brickell, T. A. \& Chatzisarantis, N. L. D. (2007). Using self-determination theory to examine the motivational correlates and predictive utility of spontaneous exercise implementation intentions. Psychology of Sport and Exercise, 8, 758-770.

BUDDEN, J. S. \& SAGARIN, B. J. (2007). Implementation intentions, occupational stress, and the exercise intention-behavior relationship. Journal of Occupational Health Psychology, 12, 391-401.

DE NOOIJER, J., DE Vet, E., BRUG, J. \& DE VRIES, N. K. (2006). Do implementation intentions help to turn good intentions into higher fruit intakes? Journal of Nutrition Education and Behavior, 38, 25-29.

ELLIOTT, M. A. \& ARMitAGE, C. J. (2006). Effects of implementation intentions on the self-reported frequency of drivers' compliance with speed limits. Journal of Experimental Psychology Applied, 12, 108-117.

GAWRILOW, C. \& GOLLWITZER, P. M. (2008). Implementation intentions facilitate response inhibition in ADHD children. Cognitive Therapy and Research, 32, 261-280.

GOLLWITZER, P. M. (1993). Goal achievement: The role of intentions. European Review of Social Psychology, 4. 141-185.

GollwITZER, P. M. (1999). Implementation intentions. Strong effects of simple plans. American Psychologist, 54, 493-503.

GOLLWITZER, P. M., BAYER, U. C. \& MCCULLOCH, K. C. (2005). The control of the unwanted. En J. A. Bargh, J. Uleman \& R. Hassin (Eds.), Unintended thought (Vol. 2, pp. 485-515), Nueva York: Guilford Press.

GOLLWITZER, P. M. \& BRANDSTÄTTER, V. (1997). Implementation intentions and effective goal pursuit. Journal of Personality and Social Psychology, 73, 186-199.

GolLWITZER, P. M. \& MosKowITZ, G. B. (1996). Goal effects on action and cognition. En E. T. Higgins \& A. W. Kruglanski (Eds.), Social psychology. Handbook of basic principles (pp. 361-399). Nueva York: Guilford Press.

GOLLWITZER, P. M. \& SCHAAL, B. (1998). Metacognition in action: The importance of implementation intentions. Personality and Social Psychology Review, 2, 124-136.

GOLLWITZER, P. M. \& SHEERAN, P. (2006). Implementation intentions and goal achievement: A meta-analysis of effects and processes. Advances in Experimental Social Psychology, 38, 69-119.

Gratton, L., POVEY, R. \& Clark-CARTER, D. (2007). Promoting children's fruit and vegetable consumption: Interventions using the theory of planned behaviour as a framework. British Journal of Health Psychology, 12, 639-650.

Henderson, M. D., Gollwitzer, P. M. \& Oettingen, G. (2007). Implementation intentions and disengagement from a failing course of action. Journal of Behavioral Decision Making, 20, 81-102.

Higgins, A. \& CONNER, M. (2003). Understanding adolescent smoking: the role of the Theory of Planned Behaviour and implementation intentions. Psychology, Health \& Medicine, 8, 173-186.

Hill, C., Abraham, C. \& Wright, D. B. (2007). Can theory-based messages in combination with cognitive prompts promote exercise in classroom settings? Social Science $\mathcal{E}$ Medicine, 65, 1049-1058.

Jackson, C., Lawton, R., Knapp, P., Raynor, D. K., Conner, M., Lowe, C. et al. (2005). Beyond intention: do specific plans increase health behaviours in patients in primary care? A study of fruit and vegetable consumption. Social Science $\mathcal{E}$ Medicine, 60, 2383-2391.

Jackson, C., LAWton, R., Raynor, D. K., Knapp, P., Conner, M., Lowe, C. et AL. (2006). Promoting adherence to antibiotics: A test of implementation intentions. Patient Education and Counseling, 61, 212-218. 
KelLar, I. \& Abraham, C. (2005). Randomized controlled trial of a brief research-based intervention promoting fruit and vegetable consumption. British Journal of Health Psychology, 10, 543-558.

KWAK, L., KREMERS, S., vAN BAAK, M. A. \& BRUG, J. (2007). Formation of implementation intentions promotes stair use. American Journal of Preventive Medicine, 32, 254-255.

Latimer, A. E., MARTin Ginis, K. A. \& Arbour, K. P. (2006). The efficacy of an implementation intention intervention for promoting physical activity among individuals with spinal cord injury: A randomized controlled trial. Rehabilitation Psychology, 51, 273-280.

Lavin, D. \& Groarke, A. (2005). Dental floss behaviour: A test of the predictive utility of the Theory of Planned Behaviour and the effects of making implementation intentions. Psychology, Health $\&$ Medicine, 10, 243-252.

LENGFELDER, A. \& GOLLWITZER, P. M. (2001). Reflective and reflexive action control in patients with frontal brain lesions. Journal of Personality and Social Psychology, 81, 80-100.

LIU, L. L. \& PARK, D. C. (2004). Aging and medical adherence: The use of automatic processes to achieve effortful things. Psychology and Aging, 19, 318-325.

LUSZCZYNSKA, A. (2006). An implementation intentions intervention, the use of a planning strategy, and physical activity after myocardial infarction. Social Science \& Medicine, 62, 900-908.

LuszCZYNSKA, A., SCHOLZ, U. \& SUTTON, S. (2007). Planning to change diet: A controlled trial of an implementation intentions training intervention to reduce saturated fat intake among patients after myocardial infarction. Journal of Psychosomatic Research 63, 491-497.

LuSZCZYNSKA, A., SOBCZYK, A. \& ABRAHAM, C. (2007). Planning to lose weight: Randomized controlled trial of an implementation intention prompt to enhance weight reduction among overweight and obese women. Health Psycbology, 26, 507-512.

MADDEN, T. J., ElLEN, P. S. \& AJZEN, I. (1992). A comparison of the theory of planned behavior and the theory of reasoned action. Personality and Social Psychology Bulletin, 18, 3-9.

Milne, S., Orbell, S. \& SheERAN, P. (2002). Combining motivational and volitional interventions to promote exercise participation: Protection motivation theory and implementation intentions. British Journal of Health Psychology, 7, 163-184.

Morrison, V. \& Bennett, P. (2008). Psicología de la salud. Madrid: Pearson.

Murgraff, V., Abraham, C. \& MCDermott, M. (2007). Reducing Friday alcohol consumption among moderate, women drinkers: Evaluation of a brief evidence-based intervention. Alcohol and Alcoholism, 42, 37-41.

Orbell, S., Hodgkins, S. \& SHEERAN, P. (1997). Implementation intentions and the theory of planned behavior. Personality and Social Psychology Bulletin, 23, 945-954.

Orbell, S. \& SheERAN, P. (2000). Motivational and volitional processes in action initiation: A field study of the role of implementation intentions. Journal of Applied Social Psychology, 30, 780-797.

Prestwich, A., Conner, M., Lawton, R., Bailey, W., Litman, J. \& Molyneaux, V. (2005). Individual and collaborative implementation intentions and the promotion of breast self-examination. Psychology \& Health, 20, 743-760

Prestwich, A., LAWTON, R. \& CONNER, M. (2003). The use of implementation intentions and the decision balance sheet in promoting exercise behavior. Psychology \& Health, 18, 707-721.

Rhodes, R. E., BlANChARD, C. M., MATHESON, D. H. \& Coble, J. (2006). Disentangling motivation, intention, and planning in the physical activity domain. Psychology of Sport and Exercise, 7, 15-27.

Rise, J., Thompson, M. \& Verplanken, B. (2003). Measuring implementation intentions in the context of the theory of planned behavior. Scandinavian Journal of Psychology, 44, 87-95.

RUTTER, D. R., STEADMAN, L. \& QUINE, L. (2006). An implementation intentions intervention to increase uptake of mammography. Annals of Behavioral Medicine, 32, 127-134.

SCHWeiger GALlo, I. \& GollWITZER, P. M. (2007). Implementation intentions: Control of fear despite cognitive load. Psicothema, 19, 280-285.

Schweiger Gallo, I., Keil, A., Mc Culloch, K. C., Rockstroh, B. \& Gollwitzer, P. M. (2009). Strategic automation of emotion regulation. Journal of Personality and Social Psychology, 96, 11-31.

SHEERAN, P. (2002). Intention-behavior relations: A conceptual and empirical review. En W. Stroebe \& M. Hewstone (Eds.), European Review of Social Psychology (Vol. 12, pp. 1-30). Nueva York: Wiley.

SHEERAN, P., Aubrey, R. \& KelLETt, S. (2007). Increasing attendance for psychotherapy: Implementation intentions and the self-regulation of attendance-related negative affect. Journal of Consulting and Clinical Psychology, 75, 853-863

Sheeran, P., Milne, S., WebB, T. L. \& Gollwitzer, P. M. (2005). Implementation intentions. En M. Conner \& P. Norman (Eds.), Predicting Health Behaviour: Research and Practice with Social Cognition Models (2 ${ }^{\text {nd }}$ ed., pp. 276-323). Buckingham: Open University Press.

SHEERAN, P. \& ORBELL, S. (1998). Does intention predict condom use? A meta analysis and test of four moderators. British Journal of Social Psychology 37, 231-250.

SHEERAN, P. \& ORBELL, S. (1999). Implementation intentions and repeated behavior: Augmenting the predictive validity of the theory of planned behavior. European Journal of Social Psychology, 29, 349-369.

SheERAN, P. \& OrbelL, S. (2000). Using implementation intentions to increase attendance for cervical cancer screening. Health Psychology, 19, 283-289.

SHEERAN, P. \& SILVERMAN, M. (2003). Evaluation of three interventions to promote workplace health and safety: evidence for the utility of implementation intentions. Social Science \& Medicine, 56, 2153-2163

SheERAN, P., WebB, T. L. \& GollWITZER, P. M. (2005). The interplay between goal intentions and implementation intention. Personality and Social Psychology Bulletin, 31, 87-98.

STEADMAN, L. \& QUINE, L. (2004). Encouraging young males to perform testicular self-examination: A simple, but effective, implementation intentions interventions. British Journal of Health Psychology, 9, 479-487.

Sullivan, H. W. \& Rothman, A. J. (2008). When planning is needed: Implementation intentions and attainment of approach versus avoidance health goals. Health Psychology, 27, 438-444.

Svenson, G. R., Östergren, P.-O., MerLo, J. \& RÁstam, L. (2002). Action control and situational risks in the prevention of HIV and STIs: Individual, dyadic, and social influences on consistent condom use in a university population. AIDS Education and Prevention, 14, 515-531.

VeRPLANKEN, B. \& FAES, S. (1999). Good intentions, bad habits, and affects of forming implementation intentions on health eating. European Journal of Social Psychology, 29, 591-604. 
Walsh, J. J., DA FonseCA, R. S. \& BANTA, A. (2005). Watching and participating in exercise videos: A test of the theory of planned behaviour, conscientiousness, and the role of implementation intentions. Psychology E Health, 20, 729-741.

WebB, T. L. \& SHEERAN, P. (2003). Can implementation intentions help to overcome ego-depletion? Journal of Experimental Social Psychology, 39, 279-286.

Wenzlaff, R. M. \& Wegner, D. M. (2000). Thought suppression. Annual Review of Psychology, 1, $59-91$.

Wiers, R. W., Cox, W. M., Field, M., Fadardi, J. S., Palfai, T. P., Schoenmakers, T. ET al. (2006). The search for new ways to change implicit alcohol-related cognitions in heavy drinkers. Alcobolism: Clinical and Experimental Research, 30 , $320-331$.

Ziegelmann, J. P., Luszczynska, A., LipPKe, S. \& SCHWARZer, R. (2007). Are goal intentions or implementation intentions better predictors of health behavior? A longitudinal study in orthopedic rehabilitation. Rehabilitation Psychology, 52, 97102 